\title{
Análisis de la situación actual de los núcleos de apoyo contable y fiscal (NAF), durante la pandemia por covid-19, en la ciudad de Quito
}

\author{
Andrés Patricio Rivadeneira Moncada \\ arivadeneira@istlnd.edu.ec \\ https://orcid.org/0000-0003-3269-6527 \\ Viviana Rocío Conrado Armas \\ vivianaconr@gmail.com \\ https://orcid.org/0000-0001-6124-9035
}

Instituto Superior Tecnológico Luis Napoleón Dillon

Quito - Ecuador

\section{RESUMEN}

La información presentada en este documento se realizó con base en una revisión bibliográfica que aborda temas como: la cultura tributaria en los ecuatorianos, obligaciones e infracciones tributarias, entre otros, lo cual permitió visualizar de manera más amplia la realidad actual de los núcleos de apoyo contable y fiscal presentes en varias IES del país. Antes y durante la pandemia por COVID-19, gran cantidad de personas se vieron en la necesidad de crear sus propias fuentes de empleo a través de la creación de emprendimientos y microempresas, diversas actividades que para ser reguladas y legales, debían inscribirse en el RUC, lo cual conlleva al cumplimiento de obligaciones y responsabilidades que no todos los contribuyentes desempeñan adecuadamente, sea por desconocimiento o por desinterés, lo cual implica un perjuicio económico no solo para el país, sino para las personas naturales y microempresas que se ven afectadas con sanciones en consecuencia de este incumplimiento. Si bien es cierto, el acceso a una asesoría contable y/o tributaria de calidad, se relaciona directamente con el pago de un valor monetario a un profesional a fin de cumplir adecuadamente, pero muchas personas desconocen que existe la posibilidad de acudir a los NAF que brindan este servicio de manera gratuita en varias IES a nivel nacional. Con la colaboración de varios docentes responsables de los NAF. se logró determinar cuáles fueron los mecanismos utilizados para que la labor de estudiantes y docentes en estos núcleos de apoyo no se detengan.

Palabras clave: tributación; impuestos; núcleos de apoyo fiscal. 


\title{
Analysis of the current situation of the accounting and tax support center (NAF), during the covid-19 pandemic, in Quito
}

\begin{abstract}
The information presented in this document was made based on a bibliographic review that addresses topics such as: tax culture in Ecuadorians, tax obligations and offenses, among others, which allowed a broader view of the current reality of the Tax Support Center present in several educative Institutions in the country. Before and during the COVID-19 pandemic, a large number of people found it necessary to create their own sources of employment through the creation of enterprises and micro-businesses, various activities that, in order to be regulated and legal, had to register in the RUC, which leads to the fulfillment of obligations and responsibilities that not all taxpayers perform adequately, either due to ignorance or lack of interest, which implies economic damage not only for the country, but also for individuals and micro-businesses that are affected by sanctions. as a consequence of this breach. While it is true, access to quality accounting and / or tax advice is directly related to the payment of a monetary value to a professional in order to comply properly, but many people are unaware that there is the possibility of going to those centers that provide this service for free in educative institutions nationwide. With the collaboration of several teachers responsible for the NAF, it was possible to determine what were the mechanisms used so that the work of students and teachers in these support centers did not stop.
\end{abstract}

Keywords: taxes; taxation; tax support center

Artículo recibido: 02 noviembre. 2021 Aceptado para publicación: 28 noviembre 2021

Correspondencia: arivadeneira@istlnd.edu.ec Conflictos de Interés: Ninguna que declarar 


\section{INTRODUCCIÓN}

Los estados dependen de la recaudación de tributos para cumplir con la obligación de brindar a la población: educación, salud, seguridad, justicia, entre otros, sin embargo, los ciudadanos ecuatorianos tienen desconfianza que sus pagos sean revertidos en obras que los beneficien, quizás porque no les interesa realizar trámites que resultan complejos y que generalmente implican la obligatoriedad de pagar impuestos, por ello su falta de cultura tributaria (Sarmiento Bayas, 2014)

A partir del año 2000, Ecuador empezó un cambio radical en su reforma tributaria, el cual estaba encaminado a reducir la evasión incrementando controles y además socializando la normativa. (Sarmiento Bayas, 2014)

"Según el SRI, la recaudación eficiente se basa en el surgimiento de una cultura tributaria que permite la realización de obras a favor de la población, sobretodo en viabilidad, salud, y educación" (Quintanilla, 2012, p.108). Independientemente del COVID-19, las personas no cumplen adecuadamente con sus obligaciones tributarias, sin embargo, la pandemia puede ser una excusa más para evadir sus responsabilidades como contribuyentes.

Para una persona natural o microempresa, el pensar en contar con una asesoría contable o tributaria de calidad, inmediatamente lo puede relacionar con "tener que desembolsar dinero", gastar recursos de los que no dispone, por ello cumplir con las obligaciones que tener un RUC implica queda en segundo plano, incluso, será difícil que conozca siquiera que existen NAF en algunas IES y concurra a estos centros para recibir el apoyo que necesita de manera gratuita.

En países como Colombia, específicamente en la Universidad Cooperativa de Colombia Sede Pereira, han optado por seguir con el funcionamiento de los NAF durante la pandemia por COVID-19. (Gutiérrez Ocampo, 2020)

A continuación, se mencionan varios casos, en los que se evidencia el trabajo de estudiantes en diferentes NAF en el año 2020 durante la pandemia. Se puede destacar, en el trabajo de Daniela Gutiérrez, con título: Núcleos de apoyo contable y fiscal, en el cual se realizaron asesorías telefónicas y a través de mensajes de Whatsapp a contribuyentes un día a la semana, para aclarar inquietudes en temas como: facturación electrónica, Régimen Único Tributario (RUT), servicios en línea. (Gutiérrez Ocampo, 2020) 
Angie Carmona, en su Informe final del Núcleo de apoyo contable y fiscal-NAF, menciona que: se atendió a las personas por llamadas telefónicas y vía Whatsapp en los cuales se brindó apoyo en los siguientes temas: Inscripción y actualización de RUT, facturación electrónica, firma electrónica, devolución de IVA (Carmona Ceballos, 2020). En el caso de Ecuador, la Universidad Técnica Particular de Loja, en su página web publica la Atención Virtual del Núcleo de Apoyo Contable Fiscal NAF - UTPL, el cual brinda asistencia virtual gratuita en aspectos contables y tributarios a través de la plataforma Zoom y con diferentes horarios de lunes a sábado (Universidad Técnica Particular de Loja, 2020).

Es el objetivo general de este estudio el elaborar un estudio sobre la situación actual de los NAF en ciertas Instituciones de Educación Superior de la ciudad de Quito, mediante el uso de instrumentos de investigación, a fin de conocer cómo ha sido el funcionamiento de estos centros durante la pandemia por COVID-19.

Dentro de la sociedad ecuatoriana, en el transcurso del tiempo la cultura tributaria se ha visto cada vez más afectada, las actividades económicas y los contribuyentes incrementan, pero así también su falta de cumplimiento ya sea como microempresarios o como personas naturales no obligadas a llevar contabilidad, debido a que en su mayoría, no cuentan con los recursos económicos para beneficiarse de una asesoría adecuada que les permita cumplir con sus obligaciones y evitar sanciones a consecuencia del desconocimiento de las leyes.

Han sido varios los mecanismos que ha implementado la Administración Tributaria para solventar las dudas de los contribuyentes como son: SRI en línea (a través de su página web), SRI telefónico (llamando al1700 SRI SRI), SRI presencial (con centros de atención y brigadas móviles), sin embargo, el menos conocido son los NAF.

Estos núcleos de apoyo funcionan en varias IES dentro del territorio nacional, y cuentan con convenios interinstitucionales en los que docentes y estudiantes de los últimos niveles son capacitados por el SRI para brindar una asesoría de calidad a las personas que lo necesiten.

Una vez que el COVID-19 fue declarado como pandemia por la OMS en marzo de 2020, en Ecuador las actividades económicas de personas naturales no obligadas a llevar contabilidad y/o microempresarios, incrementaron evidentemente. "La pandemia causada por el virus SARS -CoV-2 (Covid-19), ha ocasionado la inserción de nuevos 
emprendimientos impulsados por la desalentadora situación socio-económica en el país" (Rodríguez-Aguilar, 2020).

Pese a la declaración del Estado de Excepción y la radicalización de medidas para evitar los contagios, las personas siguieron trabajando de manera independiente para poder satisfacer las necesidades propias y de sus familias, sin embargo sus obligaciones tributarias iban quedando de lado por el desconocimiento y la falta de asesoramiento, esto no le exime de culpabilidad al contribuyente en caso de que le impongan alguna sanción, tal como lo evoca el Código Tributario en su artículo 312: "Se presume de derecho que las leyes penales tributarias son conocidas de todos. Por consiguiente, nadie puede invocar su ignorancia como causa de disculpa, salvo el caso de que la transgresión de la norma obedezca a error, culpa o dolo, o a instrucción expresa de funcionarios de la administración tributaria." (CÓDIGO TRIBUTARIO, 2018)

Los mecanismos que se implementaron para continuar con actividades laborales, educativas, comerciales, entre otras, fueron diversos, la tecnología tuvo un papel muy importante en estos acontecimientos, porque todo se trataba de realizar desde casa, el internet debía haberse considerado un servicio básico, tanto como lo son la energía eléctrica o el agua potable.

La presente investigación, busca realizar un análisis del funcionamiento de los NAF ubicados dentro de la ciudad de Quito durante el segundo semestre de 2020, en el cual, la situación del país y el mundo trataban de normalizarse paulatinamente en la mayoría de actividades posibles, brindar apoyo a los contribuyentes no estaba por demás, pero las IES eran las encargadas de tomar la iniciativa en este sentido y ver cómo realizar las asesorías gratuitas de manera efectiva durante el periodo de pandemia, así continuar con la labor para la que fueron creadas pese a la difícil situación.

El presente proyecto pretende recopilar la información necesaria del funcionamiento de los NAF en las IES de la ciudad de Quito durante el periodo de pandemia por COVID19.

Se utilizaron instrumentos de investigación, en este caso entrevistas, dirigidas a los representantes de los NAF en las IES y de ser posible encuestas a estudiantes de las mismas, para conocer sus experiencias y el trabajo que han desempeñado, teniendo en cuenta que ese tiempo es parte de sus horas de vinculación con la comunidad, además, el 
aporte del SRI como parte importante de los convenios de cooperación institucional para con las IES que cuentan con NAF.

Utilizar herramientas tecnológicas para recopilar información, es una gran ventaja, debido a que implica una inversión económica baja, ya que los recursos necesarios son internet y computador para desarrollar este paso, se evita la movilización y costos por impresiones o copias.

Los NAF están presentes en 671 universidades de 12 países que son: Argentina, Bolivia, Brasil, Chile, Colombia, Costa Rica, Ecuador, Guatemala, México, Perú, Paraguay y Honduras.

En el estudio denominado, El potencial de los Núcleos de Apoyo Contable y Fiscal, como palanca de inclusión social, cabe mencionar lo siguiente: (...) los NAF son una iniciativa muy joven y un auténtico laboratorio de innovación social. Se adaptan a las nuevas realidades como la digitalización, los desafíos de la pandemia del COVID-19, las vulnerabilidades sociales en relación con el pago de los impuestos o el reto de llegar de manera efectiva a la comunidad con un enfoque diferenciado, cumpliendo un papel social. (EUROSOCIAL, 2021)

Y si bien es cierto, este desafío tomó por sorpresa a toda la población mundial, con lo cual, la utilización de medios tecnológicos, fue una alternativa, inesperada, pero muy útil, que permitió que la mayoría de personas pueda realizar muchas actividades en el ámbito: comercial, laboral, educativo, etc., y el asesoramiento a los contribuyentes, no podía quedar de lado.

Alternativas como el uso de mensajes por redes sociales como Facebook, chats en aplicaciones como WhatsApp, videos demostrativos, han sido varios de los instrumentos que han utilizado los NAF a fin de seguir con su labor de prestar asesoría contable y tributaria de calidad, en algunos países que se encuentran integrados a esta red.

Algunos países de la región han creado incluso plataformas virtuales de intercambio para los NAF, como es el caso de la comunidad de aprendizaje para coordinadores y alumnos de la Rede NAF en Brasil (www.cuboz. com/naf) o el canal de vídeos NAF en Perú (www.sunat.tv/\#), que están operando también como repositorios y difusores de los nuevos recursos telemáticos de asesoramiento a grupos vulnerables. (EUROSOCIAL, 2021) 
En este sentido, algunos países tuvieron la iniciativa de realizar conversatorios para intercambiar experiencias de la atención de NAF durante la pandemia. La acelerada digitalización de los NAF constituye un fenómeno lleno de contradicciones que amerita ser analizado con detenimiento. Por un lado, constituye un nuevo eje tractor de desigualdad y exclusión social y, al mismo tiempo, se revela una herramienta eficaz para customizar los servicios (a las necesidades de los grupos vulnerables), así como para mejorar los dispositivos institucionales de las redes NAF. (EUROSOCIAL, 2021)

Así mismo, Daysi López, en su documento, Núcleos de Apoyo Contable y Fiscal NAF 7.0, explica que la metodología que se aplicó en los Núcleos de Apoyo Contable y Fiscal -NAF 7.0 se describe inicialmente como asesorías presenciales prestadas a los contribuyentes que se acercarían al punto de atención NAF que se encuentra ubicado las instalaciones de la Universidad Cooperativa de Colombia sede Pereira, en los días viernes en el horario comprendido desde las 3:00 pm hasta las 7:00 pm desde el 2 marzo hasta 3 julio de 2020. El horario antes descrito se cumplió en las dos primeras semanas del 2 al 13 de marzo de 2020. Después debido la emergencia decretada por el COVID19 y las medidas adoptadas por el gobierno nacional de confinamiento obligatorio, desde al 16 marzo de 2020, las asesorías se siguieron brindando de forma remota a través de WhatsApp o vía telefónica en los días jueves de 3:00 pm a 6:00 pm a los contribuyentes quienes buscaban gratuitamente orientación u acompañamiento a dudas presentadas en cuanto al cumplimiento de sus obligaciones ante la DIAN. (López Marín, 2020)

Al revisar los casos anteriormente expuestos, se puede identificar, que, en algunos NAF, la pandemia, ha sido una traba, mas no una limitante cuando de prestar asesoría tributa se trata, las personas requieren de este servicio, particularmente cuando son conscientes de que hay obligaciones por cumplir con la AT. Además, establecer horarios de atención es una manera adecuada de educar a los contribuyentes para que soliciten información en los momentos adecuados.

La cultura tributaria en Ecuador tiene varios puntos de vista entre ellos si es que pagar impuestos es un desmedro a su autoestima o a su patrimonio sin ninguna clase de la retribución o que simplemente no sepa de impuestos por costumbre o idiosincrasia. Por tanto, para que Ecuador inicia su camino hacia una adopción de una verdadera cultura tributaria la concientización del real uso del pago de impuestos debe ser parte de la educación y formación de la ciudadanía. (Quintanilla, 2012) 
La tributación es el pilar que sostiene al Estado y para poder cumplir con su obligación constitucional de velar por el bien común y proporcionar a la población los servicios básicos requeridos, necesita de recursos que provienen en gran parte de los tributos que pagan los contribuyentes. Este cumplimiento tributario puede llevarse a cabo utilizando la coerción o apelando a la razón. La fuerza se manifiesta en las leyes y su cumplimiento obligatorio, mientras que el razonamiento sólo puede darse a través de una cultura tributaria con sólidas bases. (Quintanilla, 2012)

Uno de los objetivos de la educación debe ser, romper el círculo vicioso de la discrecionalidad en el pago de tributos y hacer conciencia de que la tributación no sólo es una obligación legal, sino un deber de cada ciudadano. (Quintanilla, 2012)

Teniendo en cuenta que la Constitución se refiere a la persona como titular de derechos y obligaciones, se puede definir la cultura tributaria como el conjunto de costumbres y hábitos individuales y colectivos que permiten cumplir los deberes y defender los derechos relacionados con los tributos que se pagan al estado (Quintanilla, 2012).

Si bien es cierto existe una brecha amplia entre el Estado y los ciudadanos por la recaudación de impuestos y su destino, ese pudiera ser el inicio del cometimiento de infracciones por parte de los contribuyentes.

Las sociedades que tienen valores los cuales benefician el pacto social y los reflejan en los sistemas fiscales porque mejoran los servicios, contribuyen con su progreso económico, tecnológico y competitividad. Pero si los valores que prevalecen, no consiguen luchar contra fenómenos como la evasión fiscal, va a debilitar el tejido social y hará que se produzcan retrocesos en la cohesión social que conllevan problemas estructurales más complejos. (Bedoya, 2010)

La relación Estado-Ciudadanos no es armónica, requiere de un proceso en el que los ciudadanos conozcan y puedan manifestar su confianza en la política fiscal del Estado. Este proceso denominado legitimación, es más importante cuando existe la percepción de que el pago que realizan los ciudadanos por sus tributos, no es equivalente a los bienes y servicios que reciben del Estado. (Bedoya, 2010)

El desconocimiento de las normas tributaria o altos costos asociados al pago de impuestos que se origina por la complejidad de las leyes, pueden no sólo iniciar el camino hacia la evasión, sino además generan resentimientos que debilitan la conciencia moral de los 
contribuyentes lo que conlleva a la evasión como forma de resarcimiento con la AT. (Bedoya, 2010)

Según varios autores se considera que la cultura tributaria es una de las tantas, sino la principal causa de no pago de impuestos por evasión elusión otros ilícitos tributarios dejando aparte la de la defraudación tributaria ya que está tiene ciertas trazas de dolo. La administración tributaria en general está inmersa en esta falta de cultura tributaria ya que los sistemas de recaudación tributaria son poco eficientes y arcaicos, además de implementar altos costos indirectos al momento de realizar el pago de impuestos sin olvidarse de las complicadas reglas existentes y aplicables al tratar de cumplir deberes formales. Esto causa un cierto desagrado del ciudadano al pagar impuestos. (Mendoza, 2016)

El Código Tributario ecuatoriano, en su artículo 15, conceptualiza a la Obligación Tributaria como "El vínculo jurídico personal, existente entre el Estado o las entidades acreedoras de tributos y los contribuyentes o responsables de aquellos, en virtud del cual debe satisfacerse una prestación en dinero, especies o servicios apreciables en dinero, al verificarse el hecho generador previsto por la ley.” (CÓDIGO TRIBUTARIO, 2018)

En el trabajo de Santillana (2013), se define como evasión fiscal a toda acción u omisión parcial o total, tendiente a reducir o retardar el cumplimiento de la obligación tributaria. La evasión no sólo implica una pérdida de ingresos fiscales, con sus efectos en la prestación de servicios públicos, sino que afecta la equidad de los impuestos.

Además, en el caso de los impuestos fiscales la evasión es el delito de tratar de evitar con alguna artimaña pagar las obligaciones fiscales. (Santillana, 2013)

La evasión tributaria es el no pago de la obligación tributaria, lo que implica una sanción establecida en la legislación, debido a que se aprecia medianamente el incumplimiento de la obligación tributaria. El ejemplo más frecuente es en el que las personas a fin de no pagar tributos o pagarlos en menor cantidad, oculta parcial o totalmente los hechos que dan origen a la obligación tributaria. (Echaiz Moreno, 2014)

Con el fin de verificar si la elusión tributaria nace de la falta de cultura tributaria es necesario precisar este tipo de ilícito. García, (2005) lo define cómo la búsqueda de fórmulas menos onerosas desde el punto de vista fiscal a través de alternativas negociales qué provocan una reducción del tributo, Por su lado, Echaiz Moreno, (2014) lo conceptualiza como una actividad de un sujeto direccionada a eliminar ciertas 
obligaciones tributarias principales mediante figuras contratos negocios otros actos qué son perfectamente legales y se llevan a cabo en realidad de los hechos, este autor no lo define como algo malo o bueno, mientras que Tipke, (2002) desde otro punto de vista indica qué es una actividad consciente y planificada que da como resultado una resistencia fiscal legal que no es inmoral, pero qué causa problemas a los Estados de Derecho que respetan la libertad.

No profundizaremos sobre la idea de ilícito tributario o de formación tributaria ya que este es un acto típico, antijurídico y sancionado por una pena que cumple condiciones de punibilidad (Siguencia, 2009) teniendo la característica de existir dolo, causando que la cultura tributaria no sea una causa de peso para cometer este tipo de delitos, bajo la idea de qué para que haya dolo, debe haber una intención de dañar mediante sus actos y no un mero desconocimiento de la normativa vigente.

Los núcleos de apoyo contable y fiscal son centros qué se dedican a dar información sobre temas principalmente tributarios, aunque también lo hacen de índole contable por parte de estudiantes de carreras que pueden ser bien administrativas contables y tributarias de manera gratuita a personas naturales no obligadas a llevar contabilidad y a microempresarios esto siempre bajo la supervisión de docentes universitarios (Servicio de Rentas Internas, 2020)

Los NAF promueven la interacción entre la Administración Tributaria (AT), las IES, los alumnos y la sociedad, y propicia mediante la cooperación mutua la cualificación de los futuros profesionales contables y la prestación de servicios fiscales a contribuyentes desfavorecidos económicamente, a fin de reforzar la imagen de ambos ante la sociedad y de desarrollar la conciencia tributaria y la ciudadanía. (RED NAF, 2016)

La Receita Federal de Brasil en 2011 diseñó estos NAF siendo efectivamente implementados en 300 universidades de este país por otro lado EUROSOCIAL, Ha impulsado desde 2014 el avance de este tipo de núcleos Ya que esto logra intercambio de información entre las administraciones tributarias y las universidades indistintamente del país que se trate.

Se han utilizado diversas herramientas como: talleres, asesorías especializadas, guías y manuales. Todas las actuaciones han estado presididas por un diálogo participativo con los docentes, los responsables universitarios y los estudiantes, con objeto de socializar la 
idea y consensuar los pasos a seguir para su adaptación nacional por parte de los propios actores involucrados. (EUROSOCIAL, 2018)

Brasil ha liderado este proceso. Sin embargo, a medida que otros países han avanzado en la creación de los NAF, han ido aportando sus modelos de capacitación y de convenio, así como las experiencias directas de funcionarios, docentes y estudiantes, o la utilización de las nuevas tecnologías. (EUROSOCIAL, 2018)

Los servicios prestados por los NAF se han orientado mayoritariamente a las personas físicas de bajos recursos que no tienen acceso a servicios de asesoría contable y fiscal. Como norma general, este perfil de usuarios/as se corresponde con personas microemprendedoras que gestionan micronegocios, con diferentes grados de formalización, y que se ven sometidos a elevados grados de vulnerabilidad económica y desprotección social.

Los NAF han definido este perfil de usuarios a través de un amplio abanico de descriptores genéricos: microemprendedores, trabajadores independientes, cuentapropistas y, en ocasiones, también con denominaciones sectorializadas, tales como comerciantes minoristas, tenderos, vendedores ambulantes, artesanos, etc. Esta riqueza de descriptores no es sino una expresión de un fenómeno latinoamericana: la enorme prevalencia del sector informal o popular de la economía. (EUROSOCIAL, 2021)

Es importante recalcar que los NAF no son un puesto de atención al contribuyente de la AT; no es un sustituto de los despachos de contabilidad o de asesoría fiscal y tampoco necesariamente un convenio oficial entre la AT y las instituciones educativas. (Red Núcleos de Apoyo contable y Fiscal, s/f)

Los núcleos de apoyo se utilizan principalmente como centros en dónde alumnos pueden realizar las horas de vinculación con la comunidad Existentes en la normativa vigente de educación superior.

La vinculación con la comunidad es una función sustantiva de educación superior orientada a integrar a los estudiantes con su entorno con el fin de apoyar A las otras funciones sustantivas como investigación y docencia a través de planes programas y proyectos formulados Manera sistemática y multidisciplinaria esto con el fin de fortalecer la creación de soluciones viables e innovadoras a las problemáticas de la comunidad (UTE $\mathrm{EC}, 2017)$ 
Ahora bien, estos Núcleos tienen la particularidad de qué son de atención presencial, por tanto, hay que tener en cuenta que a finales del año 2019 e inicios del 2020, el mundo y por ende el Ecuador, se verían afectados un virus que cambiaría de manera inesperada la vida y la normalidad tal como en algún momento se la había conocido, el inicio del COVID-19.

En febrero de 2020, autoridades del Ministerio de Salud anunciaron que Guayaquil se había convertido en escenario del primer caso confirmado del nuevo coronavirus en Ecuador. (EL COMERCIO, 2020)

La noche del lunes 16 de marzo, el presidente de la República, declaró el Estado de Excepción en todo el territorio ecuatoriano, con el propósito de contener la transmisión del COVID-19 y en virtud de la declaración de Estado de Excepción en el país, se disponen que las actividades presenciales laborales, educativas y otras más, se realicen de manera telemática causando que los NAF se cierren.

Las tecnologías digitales en el contexto de la pandemia COVID-19 han tomado relevancia al constituir, en muchos casos, el único nexo de los alumnos con las escuelas, trasladando los procesos de enseñanza al ámbito virtual. Al margen del enorme esfuerzo de muchos docentes, han quedado de manifiesto deficiencias crónicas del sistema educativo, entre ellas la baja competencia digital y, sobre todo, el aumento de la desigualdad en el acceso. (Salinas Ibáñez, 2020)

La pandemia ha puesto a prueba al sistema educativo, sus fortalezas y oportunidades ya que este sector es uno de los más afectados por la pandemia, siendo sus efectos muy evidentes. En el pico de la pandemia hasta 1500 millones de estudiantes no pudieron asistir a la escuela y en muchos casos no pueden hacerlo todavía ni siquiera por vía telemática (Salinas Ibáñez, 2020)

El aprendizaje por vía telemática se convirtió en una solución rápida, más no efectiva ya que fue aplicada, en la mayoría de casos, de una manera emergente utilizando tecnologías digitales en las cuales se aplicaron las mismas metodologías de la enseñanza presencial en los procesos en línea, causando que la enseñanza-aprendizaje tenga poca calidad. Hay que recalcar que la educación en línea no es el inconveniente, la verdadera problemática fue la utilización de metodologías erróneas en su ejecución. (Salinas Ibáñez, 2020)

De igual manera se debe reconocer las impresionantes actuaciones aplicadas por parte de muchos educadores que dieron pasó a qué la educación no se detuviera además de algunos 
sistemas de Educación nacionales que ofrecieron rápidamente su contingente con el fin de seguir con la educación mediante herramientas de conexión remota (Salinas Ibáñez, 2020)

Según Dueñas, (2020) la educación es la formación de aprendizaje qué tiene como principal proyecto formar profesionales que estén en la capacidad de generar innovaciones alineadas a las nuevas tecnologías, las cuales se deben aplicar a la vida cotidiana. Sobre esta idea se debe verificar si el alumno de esta nueva era cuenta con estas habilidades tecnológicas, y en concordancia a lo acotado por Rubio, (2020) siendo nativos digitales son capaces de usar tecnologías desconocidas con mucha naturalidad, pero esto no significa que tengan conocimientos avanzados de estas tecnologías, por tal motivo, también necesitan ser alfabetizados en el manejo de estas herramientas digitales.

Las tecnologías de la información y comunicación han sido un avance significativo en cuanto al acceso a la información gracias al internet. Esto supone un avance en la forma de educar porque se experimenta en un escenario totalmente nuevo en el ámbito formativo ya que es una herramienta muy interesante al momento del intercambio de conocimiento entre docentes y estudiantes, sobre todo en procesos en línea, permitiendo que personas que se encuentran en diferentes lugares físicos puedan realizar actividades colaborativas, reflexivas y críticas en un ambiente moderno y amigable. (Dueñas, 2020)

Ahora bien una vez indicado que la pandemia causó un aislamiento de todas las personas y que la tecnología tuvo un papel principal en que el mundo no se detenga verificaremos si las actividades realizadas por los NAF no se detuvieron ya que como lo indica Torres, (2020) una de las propuestas más importantes de la UNESCO fue trasladar al hogar las actividades escolares convirtiendo a los profesores en mediadores de esta educación a distancia ya los padres de familia en sujetos mucho más activos en la formación en la modalidad en línea que en la presencialidad, y dejando en claro la mala situación en la que se encuentra el Ecuador en equipamientos, competencias y uso de la digitalización y de las nuevas tecnologías además de las diferencias entre la educación pública y la privada

\section{ESTRATEGIAS METODOLÓGICAS O MATERIALES Y MÉTODOS}

Para desarrollar la presente investigación, se utilizó un enfoque cuantitativo, después de haber planteado el respectivo problema de investigación, construido el marco teórico, se buscó comprobar la hipótesis planteada y cuantificarla, utilizando los siguientes criterios: 
Según el proceso formal: Inductiva, que permitirá, a partir de casos particulares, obtener una conclusión general.

Según el grado de abstracción: Investigación aplicada, la cual se encamina a la resolución de problemas; según la manipulación de las variables: Descriptivo, el cual no permite la manipulación de variables; según la dimensión cronológica: Descriptivo, que refiere los fenómenos tal cual aparecen en el presente; según el objetivo: Descriptivo, el cual a partir de la observación permitirá describir el fenómeno. según el lugar: Campo, que analiza la situación de manera natural en el lugar que se produce; según la temporalización: Transversal, que permita realizar la investigación en un periodo corto de tiempo, utilizando una muestra; según el número de individuos: Estudios de grupo, mediante una muestra representativa seleccionada de la población.

Hay que tener en cuenta que se utilizó la metodología descriptiva, en la que el investigador se acerca a la realidad tratando de describir y documentar cómo son los fenómenos que en ella acontecen. Se basa, pues, en la observación que se realiza en el ambiente natural donde se producen los fenómenos. (Tejada, 1997)

El instrumento considerado para obtener los resultados en esta investigación, es la aplicación de entrevistas.

\section{Las fuentes que se van a tener en cuenta son:}

- Fuentes primarias: Bibliografía consultada

- Fuentes secundarias: Docentes responsables de los NAF

\section{RESULTADOS Y DISCUSIÓN}

Los resultados de esta investigación se obtuvieron a través de entrevistas a docentes coordinadores de los NAF de las IES a nivel nacional, para contactarse con ellos se utilizaron los correos electrónicos y contactos que se encuentran publicados en la página del SRI, de los 34 NAF en el Ecuador fue posible obtener respuesta de 8. En este caso se utilizó la herramienta ZOOM para videoconferencias, lo cual facilitó la obtención de resultados a la encuesta preparada previamente. Para procesar los resultados obtenidos se plantearon criterios de acuerdo a cada pregunta, que en su mayoría fueron preguntas abiertas.

Los docentes coordinadores de NAF que respondieron a la invitación para esta entrevista fueron:

- Universidad Central del Ecuador- Dra. Paulina Calvache 
- Pontificia Universidad Católica del Ecuador- Sede Quito- Ing. Janneth Cando

- Universidad UTE- Ing. Marco Núñez

- Pontificia Universidad Católica del Ecuador- Sede Esmeraldas- Ing. Verónica Aguilar

- Universidad Estatal Península de Santa Elena- Ing. Wilson Toro

- Universidad Católica de Cuenca- Sede Azogues- Ing. Fabián Ramírez

- Universidad Católica de Cuenca- Sede Macas- Ing. Paola Carangui

- Universidad Católica de Cuenca- Sede Cuenca- Ing. Patricio Mendieta

La iniciativa del SRI para el apoyo de implementación de NAF en las IES, comienza en 2015, a partir de este año se firman los convenios de cooperación interinstitucional con aquellas instituciones que cumplían con los requisitos necesarios para este fin. Estos convenios tienen una duración de 5 años, en varias IES estos han cumplido su vigencia y a causa de la pandemia no han podido ser renovados, adicionalmente, estudiantes y docentes son capacitados previamente por el SRI y como requisito indispensable deben aprobar un curso para su participación en los NAF.

Dentro de las condiciones para que los estudiantes de las IES obtengan su grado, es el cumplimiento de sus horas de vinculación con la sociedad, la participación en NAF les da una gran ventaja a los estudiantes de carreras administrativas, porque además de completar estas horas les permite obtener conocimiento real fuera de las aulas sobre temas contables y tributarios.

Los temas más consultados por los contribuyentes que asisten a las asesorías gratuitas que brindan los NAF, incluyen lo correspondiente a declaraciones y devoluciones de impuestos para lo cual es necesario el acceso a internet y manejo de la plataforma del SRI, recursos y conocimientos que son poco accesibles para todas las personas que tienen un RUC.

Además, hay que tener en cuenta que no todos los contribuyentes son conscientes de las obligaciones que conlleva el obtener un RUC, sin embargo, una parte de ellos llega a los NAF a pedir ayuda cuando ya tienen alguna notificación por incumplimiento, no lo hacen de manera preventiva que sería lo ideal, para evitar relacionar al SRI como una entidad creada exclusivamente para castigar al contribuyente.

Durante la pandemia, en los NAF implementaron diversos mecanismos para no solo cumplir con el objetivo para el que fueron creadas, sino también por la necesidad de los estudiantes del cumplimiento de sus horas de vinculación con la sociedad, si bien es cierto 
hubieron ventajas y limitantes, pero la labor continuó aunque los resultados no fueran siempre los esperados.

Independientemente de la situación en la que esté atravesando la sociedad, cabe destacar los beneficios que obtienen los estudiantes que participan en los NAF, el conocimiento adquirido es un aporte para su formación integral y les da una ventaja adicional, llevar a la práctica de una forma real, lo aprendido en las aulas.

Existe en la idiosincrasia de las personas la idea de que la burocracia es una limitación muy grande al momento de cumplir con las obligaciones tributarias y ya que la pandemia causó que muchas actividades que ya de por sí eran muy difíciles de realizar se complicaran aún más, los NAF cumplieron su papel al innovar y no dejarse vencer, esto con la idea de que ir a uno de estos núcleos para recibir información es y fue, más simple que en los centros de atención de las administraciones tributarias.

\section{CONCLUSIÓN Y CONSIDERACIONES FINALES}

Los NAF a nivel nacional como internacional, durante la pandemia por COVID-19 han tenido que adaptarse a la nueva normalidad, es decir al uso de herramientas tecnológicas y todo lo que ello conlleva, el distanciamiento social ha sido un factor determinante en el trabajo en los NAF, porque tanto alumnos como docentes no pueden interactuar de la misma forma, tal como se lo realizaba de manera presencial, debido por una parte a que no todos los contribuyentes cuentan con las herramientas tecnológicas adecuadas para tener una asesoría virtual apropiada, teniendo en cuenta además que muchas veces se requiere del manejo de información en documentos físicos, por ello el objetivo de asesoría tributaria y contable gratuita, no se ha cumplido en su totalidad para la gran mayoría de IES en el país, sin embargo se han adaptado, al punto de ser los participantes quienes van a buscar a los contribuyentes que requieren ayuda.

Dentro de la formación de los estudiantes que cursan carreras contables y administrativas, la participación en NAF les brinda la oportunidad de enfrentarse a la realidad y de aplicar los conocimientos adquiridos en las aulas, razón por la cual, durante esta pandemia, esa posibilidad de formación integral se ha visto afectada, porque las asesorías solicitada por los contribuyentes han disminuido, el trabajo de los participantes es menor e incluso eso conlleva a que el número de estudiantes que forman parte de los NAF semestralmente, disminuya. 
En este caso la tecnología ha jugado un papel fundamental para aquellas IES que han optado por continuar brindando asesorías a microempresarios y personas naturales no obligadas a llevar contabilidad, a partir del segundo semestre del año 2020, el uso de programas como: ZOOM para videoconferencias, correos electrónicos, Whatsapp, además de las bases de datos e incluso la búsqueda de contribuyentes fuera de las IES, han sido algunas de las alternativas adoptadas para que no se detenga el trabajo de docentes y estudiantes, después de que la mayoría de NAF dejó de funcionar de manera presencial al inicio de la pandemia en marzo de 2020.

Durante el segundo semestre de 2020, los mecanismos implementados por las IES que tienen a cargo el funcionamiento de NAF, fueron diversos, estudiantes y docentes encontraron situaciones tanto positivas como negativas, sin embargo eso no fue un limitante para continuar fomentando la cultura tributaria en los ciudadanos que lo necesitaban.

La pandemia por COVID-19 obligó a muchas empresas y personas a utilizar herramientas nuevas, de manera que las actividades cotidianas que se realizaban de manera presencial fuera factibles realizarlas desde los hogares con el apoyo de la tecnología, por ello, en la actualidad esta alternativa dual, se la puede seguir utilizando por su eficiencia y eficacia en gran cantidad de labores.

Por ultimo presentamos la recomendación de que hay que tener en cuenta que, durante la pandemia, la tecnología ha sido la clave para continuar con las actividades cotidianas, por ende, una mejor capacitación para docentes y estudiantes a fin de manejar herramientas que les ayuden a brindar asesorías virtuales considero que sería fundamental de aquí en adelante.

El acceso gratuito a internet para aquellos contribuyentes que no cuentan con recursos suficientes, ni para pagar una asesoría particular, menos para contratar planes de datos, sería un gran apoyo por parte de la empresa privada o del mismo gobierno que se beneficia cuando el ciudadano cumple adecuada y oportunamente sus obligaciones y fomenta la cultura tributaria.

La difusión por medios de comunicación masivos, sería un gran apoyo para la comunidad que desconoce muchas veces que puede recibir un servicio de asesoría gratuita y de calidad brindado por estudiantes y docentes preparados para participar en NAF. 
Finalmente, la información que proporciona el SRI debería estar actualizada en su página, tanto correos electrónicos como números telefónicos, teniendo en cuenta que es una de las alternativas para aquellas personas naturales no obligadas a llevar contabilidad y microempresarios que requieren de la asesoría que prestan los NAF.

\section{LISTA DE REFERENCIAS}

Bedoya, A. y. (julio a diciembre de 2010). Entendiendo la moral tributaria en Ecuador. Fiscalidad. Revista Institucional del Servicio de Rentas Internas, 91-132. Quito: SRI.

Carmona Ceballos, A. V. (2020). Informe final del Núcleo de apoyo contable y fiscalNAF. [tesis de pregrado, Universidad Cooperativa de Colombia]. Obtenido de Repositorio Institucional UCC: http://hdl.handle.net/20.500.12494/18452

CÓDIGO TRIBUTARIO. (21 de 08 de 2018). CÓDIGO TRIBUTARIO. CÓDIGO TRIBUTARIO. Ecuador: Registro Oficial Suplemento 38 de 14-jun.-2005.

Constitución Política de la República del Ecuador. (20 de 10 de 2008). Constitución Política de la República del Ecuador. Constitución Política de la República del Ecuador. Ecuador: Registro Oficial 449.

Dueñas, A. E. (2020). Incidencia de la tecnología en el entorno educativo del Ecuador frente a la pandemia del covid-19. Polo del Conocimiento: Revista científicoprofesional, 5(10), 754-773.

Echaiz Moreno, D. \&. (2014). La Elusión Tributaria: Análisis Crítico de la Actual Normatividad y Propuestas para una Futura Reforma. Lima: Derecho \& Sociedad, (43), 151-167. Obtenido de https://revistas.pucp.edu.pe/index.php/derechoysociedad/article/view/12567

EL COMERCIO. (29 de 02 de 2020). Ministerio de Salud confirma primer caso de coronavirus en Ecuador. Obtenido de elcomercio.com: https://www.elcomercio.com/tendencias/sociedad/salud-confirma-primer-casocoronavirus.html

EUROSOCIAL. (11 de 2018). Buenas prácticas para el desarrollo de los Núcleos de Apoyo Contable y Fiscal (NAF). COLECCIÓN EUROSOCIAL $N^{o}$ 3. Madrid, España: Programa EUROsociAL. 
EUROSOCIAL. (01 de 2021). El potencial de los Núcleos de Apoyo Contable y Fiscal (NAF) como palanca de inclusión social. COLECCIÓN EUROSOCIAL $N^{\circ} 18$. Madrid, España: Programa EUROsociAL.

Ferrer, R. (2020). Pandemia por Covid-19: el mayor reto de la historia del intensivismo. Medicina intensiva, 44 (6), 323. Obtenido de Medicina intensiva.

García, C. (2005). Reflexiones sobre las medidas para combatir la elusión fiscal. Cláusulas generales y especiales. Lima: Facultad de Derecho de la Pontificia Universidad Católica del Perú.

Gutiérrez Ocampo, D. (2020). Núcleos de apoyo contable y fiscal [Trabajo de pregrado, Universidad Cooperativa de Colombia]. Obtenido de Repositorio Institucional UCC.: http://hdl.handle.net/20.500.12494/28510

López Marín, D. V. (07 de 08 de 2020). Núcleos de apoyo contable y fiscal NAF 7.0. [Tesis de pregrado, Universidad Cooperativa de Colombia]. Repositorio Institucional UCC. Obtenido de Repositorio Institucional UCC: http://hdl.handle.net/20.500.12494/19987

Mendoza, F. P. (2016). Correlación entre cultura tributaria y educación tributaria universitaria: Caso Universidad Estatal de Sonora. Obtenido de Revista Global de Negocios. Vol.4 $\quad \mathrm{N}^{\mathrm{o}} \quad 1, \quad 61-76$ : https://papers.ssrn.com/sol3/papers.cfm?abstract_id=2659374

Quintanilla, J. (2012). La universidad en la cultura tributaria. Quito: Editorial Abya Yala.

RED NAF. (01 de 04 de 2016). MANUAL DE ESTANDARIZACIÓN DE LOS NÚCLEOS DE APOYO CONTABLE Y FISCAL EN AMÉRICA LATINA. MANUAL DE ESTANDARIZACIÓN DE LOS NÚCLEOS DE APOYO CONTABLE Y FISCAL EN AMÉRICA LATINA. RED NAF.

Red Núcleos de Apoyo contable y Fiscal. (s/f de s/f de s/f). Red Núcleos de Apoyo contable y Fiscal. Obtenido de Red Núcleos de Apoyo contable y Fiscal: http://rednaf.educacionfiscal.org/pagina/naturaleza-de-los-naf

Rodríguez-Aguilar, V. R.-P.-M.-Q. (2020). Nuevos emprendimientos: Un análisis a sus tipos y posicionamiento postcovid-19. Obtenido de Revista Científica FIPCAEC (Fomento De La investigación Y publicación En Ciencias Administrativas, Económicas Y Contables).: https://doi.org/10.23857/fipcaec.v5i4.298 
Romero, M. (17 de 01 de 2007). Aspectos Legales Empresariales. Obtenido de Aspectos Legales Empresariales: https://nella-romero.blogspot.com/2007/01/ilicitostributarios.html

Rubio, A. Á. (2020). Jóvenes y generación 2020. Revista de estudios de juventud, 11.

Salinas Ibáñez, J. (2020). Educación en tiempos de pandemia: tecnologías digitales en la mejora de los procesos educativos. Revista Innovaciones Educativas, 17-21.

Santiana, A. (2013). Medidas tendientes a combatir la elusión y la evasión fiscal de impuesto a la renta de las sociedades en el Ecuador. Quito: Universidad Andina Simón Bolívar, Sede Ecuador. Área de Derecho. Obtenido de http://hdl.handle.net/10644/3711

Sarmiento Bayas, C. (2014). La cultura tributaria en Ecuador. Obtenido de Observatorio de la Economía Latinoamericana: https://www.eumed.net/cursecon/ecolat/ec/2014/cultura-tributaria.html

Secretaría General de Comunicación de la Presidencia. (16 de 03 de 2020). Secretaría General de Comunicación de la Presidencia. Obtenido de Secretaría General de Comunicación de la Presidencia: https://www.comunicacion.gob.ec/elpresidente-lenin-moreno-decreta-estado-de-excepcion-para-evitar-lapropagacion-del-covid-19/

Servicio de Rentas Internas. (08 de 2013). MI GUIA TRIBUTARIA DEBERS FORMALES. MI GUIA TRIBUTARIA DEBERS FORMALES. Quito, Pichincha, Ecuador.

Servicio de Rentas Internas. (20 de 02 de 2020). Servicio de Rentas Internas. Obtenido de SRI: https://www.sri.gob.ec/web/guest/nucleos-de-apoyo-contable-y-fiscalnaf

Siguencia, L. y. (enero de 2009). El ilícito tributario en la legislación ecuatoriana. Loja, Ecuador. Obtenido de http://dspace.utpl.edu.ec/xmlui/bitstream/handle/123456789/5074/tesis.pdf?sequ ence $=1 \&$ isAllowed $=\mathrm{y}$

Tejada, J. (1997). El proceso de investigación científica. En J. Tejada Fernández , El proceso de investigación científica (págs. 72-76). Barcelona: Fundación "la Caixa". 
Tipke, K. (2002). Moral tributaria del Estado y de los contribuyentes. Madrid: Editorial Marcial Pons.

Torres, R. M. (2020). El Ecuador y la digitalización de la educación. . Obtenido de El Ecuador y la digitalización de la educación. : https://otraeducacion.blogspot.com/2020/04/el-ecuador-y-la-digitalizacion-de-la.html

Universidad Técnica Particular de Loja. (2020). Atención del Núcleo de Apoyo Contable, Fiscal NAF. Obtenido de Universidad Técnica Particular de Loja: https://www.utpl.edu.ec/carreras/atenci\%C3\%B3n-del-n\%C3\%BAcleo-deapoyo-contable-fiscal-naf

UTE EC. (s/n de $\mathrm{s} / \mathrm{n}$ de 2017). UTE EC. Obtenido de UTE EC: https://www.ute.edu.ec/vinculacion-con-la-sociedad/

\section{LISTADO DE ABREVIACIONES}

NAF Núcleo de Apoyo Contable y Fiscal

IES Instituciones de Educación Superior

RUC Registro Único de Contribuyentes

SRI Servicio de Rentas Internas

RUT Régimen Único Tributario

AT Administración Tributaria

DIAN Dirección de Impuestos y Aduanas Nacionales

OMS Organización Mundial de la Salud

COIP Código Orgánico Integral Penal

TIC Tecnologías de la Información y Comunicación

UNESCO Organización de las Naciones Unidas para la Educación, la Ciencia y la Cultura

UCE Universidad Central del Ecuador

PUCE Pontificia Universidad Católica del Ecuador

UPSE Universidad Estatal Península de Santa Elena 